\title{
Tyrosine kinase inhibitor SU6668 represses chondrosarcoma growth via antiangiogenesis in vivo
}

Frank M Klenke ${ }^{1}$, Amir Abdollahi², Elisabeth Bertl ${ }^{3}$, Martha-Maria Gebhard ${ }^{4}$, Volker Ewerbeck ${ }^{5}$, Peter E Huber ${ }^{2}$ and Axel Sckell ${ }^{* 6}$

\begin{abstract}
Address: ${ }^{1}$ Department of Orthopedic Surgery, Inselspital, University of Bern, CH-3010 Bern, Switzerland, ${ }^{2}$ Department of Radiation Oncology, German Cancer Research Center, INF 280, D69120 Heidelberg, Germany, ${ }^{3}$ Division of Toxicology and Cancer Risk Factors, German Cancer Research Center, INF 280, D-69120 Heidelberg, Germany, ${ }^{4}$ Department of Experimental Surgery, University of Heidelberg, INF 365, D-69120 Heidelberg, Germany, ${ }^{5}$ Department of Orthopedic Surgery, University of Heidelberg, Schlierbacher Landstrasse 120a, D-69118 Heidelberg, Germany and ${ }^{6}$ Department of Trauma and Reconstructive Surgery, Charité University Medical Center, Campus Benjamin Franklin, Hindenburgdamm 30, D-12200 Berlin, Germany
\end{abstract}

Email: Frank M Klenke - frank.klenke@dkf.unibe.ch; Amir Abdollahi - a.amir@dkfz.de; Elisabeth Bertl - elisabeth.bertl@gmx.net; MarthaMaria Gebhard - gebhard@exchi.uni-heidelberg.de; Volker Ewerbeck - volker.ewerbeck@ok.uni-heidelberg.de;

Peter E Huber - p.huber@dkfz.de; Axel Sckell* - axel.sckell@charite.de

* Corresponding author

Published: 17 March 2007

BMC Cancer 2007, 7:49 doi:10.1186/147|-2407-7-49
Received: 2 June 2006

Accepted: 17 March 2007

This article is available from: http://www.biomedcentral.com/I47I-2407/7/49

(C) 2007 Klenke et al; licensee BioMed Central Ltd.

This is an Open Access article distributed under the terms of the Creative Commons Attribution License (http://creativecommons.org/licenses/by/2.0), which permits unrestricted use, distribution, and reproduction in any medium, provided the original work is properly cited.

\begin{abstract}
Background: As chondrosarcomas are resistant to chemotherapy and ionizing radiation, therapeutic options are limited. Radical surgery often cannot be performed. Therefore, additional therapies such as antiangiogenesis represent a promising strategy for overcoming limitations in chondrosarcoma therapy. There is strong experimental evidence that SU6668, an inhibitor of the angiogenic tyrosine kinases Flk-I/KDR, PDGFRbeta and FGFRI can induce growth inhibition of various primary tumors. However, the effectiveness of SU6668 on malignant primary bone tumors such as chondrosarcomas has been rarely investigated. Therefore, the aim of this study was to investigate the effects of SU6668 on chondrosarcoma growth, angiogenesis and microcirculation in vivo.
\end{abstract}

Methods: In 10 male severe combined immunodeficient (SCID) mice, pieces of SWI353 chondrosarcomas were implanted into a cranial window preparation where the calvaria serves as the site for the orthotopic implantation of bone tumors. From day 7 after tumor implantation, five animals were treated with SU6668 ( $250 \mathrm{mg} / \mathrm{kg}$ body weight, s.c.) at intervals of 48 hours (SU6668), and five animals with the equivalent amount of the CMC-based vehicle (Control). Angiogenesis, microcirculation, and growth of SW 1353 tumors were analyzed by means of intravital microscopy.

Results: SU6668 induced a growth arrest of chondrosarcomas within 7 days after the initiation of the treatment. Compared to Controls, SU6668 decreased functional vessel density and tumor size, respectively, by $37 \%$ and $53 \%$ on day 28 after tumor implantation. The time course of the experiments demonstrated that the impact on angiogenesis preceded the anti-tumor effect. Histological and immunohistochemical results confirmed the intravital microscopy findings.

Conclusion: SU6668 is a potent inhibitor of chondrosarcoma tumor growth in vivo. This effect appears to be induced by the antiangiogenic effects of SU6668, which are mediated by the inhibition of the key angiogenic receptor tyrosine kinases Flk-I/KDR, PDGFRbeta and FGFRI. The experimental data obtained provide rationale to further develop the strategy of the use of the angiogenesis inhibitor SU6668 in the treatment of chondrosarcomas in addition to established therapies such as surgery. 


\section{Background}

Chondrosarcomas are the second most frequent malignant primary bone tumors in humans and are usually resistant to ionizing radiation and chemotherapy. To date, the only curative therapy is the surgical resection provided that an R0-situation can be achieved and metastases do not exist at the time of the diagnosis.

Due to the knowledge that a solid tumor cannot grow beyond a critical size of $1-2 \mathrm{~mm}^{3}$ or metastasize without an adequate blood supply $[1,2]$, therapeutic strategies targeting tumor vasculature, i.e. antiangiogenic therapies, represent a promising therapeutic option in addition to established therapies. Cells from the tumor vasculature are generally non-transformed and less prone to acquiring drug resistance [3]. Therefore, endothelial processes are considered as ideal targets for the prevention and control of tumor growth [4]. The idea of inhibiting tumor neovascularization without causing negative side-effects in the host vascular system is based further on the observation that the vasculature in normal adults is generally quiescent, with only $0.01 \%$ of endothelial cells undergoing cell division at any given time. Regarding tumor vasculature, the fraction of cycling endothelial cells may be 2-3 orders of magnitude higher. Thus, agents that antagonize active signal transduction are likely to have a minimal effect on the normal vasculature and therefore allow a more targeted approach regarding proliferating tumor vessels [5]. In principle, antiangiogenic therapy is applicable on any tumor entity, as endothelial cells represent a common cell type of all solid tumors [4]. Substances inhibiting active signal transduction of the angiogenic cascade, such as inhibitors of the receptor tyrosine kinases, have shown promising antiangiogenic effects in preclinical settings if administered as single agents or in combination with established therapies such as chemotherapy and radiation [6-12].

SU6668 is a small-molecule inhibitor of the receptor tyrosine kinases Flk-1/KDR (vascular endothelial growth factor receptor 2, VEGR2), PDGFRbeta (platelet-derived growth factor receptor beta) and FGFR1 (fibroblast growth factor receptor 1), which play an important role in angiogenesis, as they transduce the signals of the key angiogenic growth factors VEGF, PDGF and bFGF. Due to this mechanism, SU6668 is considered to be a potent substance for antagonizing central pathways of angiogenic signal transduction. The substance has shown promising antiangiogenic effects on primary tumors such as colon and lung carcinomas [9-12]. However, studies on the therapeutic efficiency of receptor tyrosine kinase inhibitors such as SU6668 on primary bone tumors are very rare [13]. The aim of the present study, therefore, was to investigate the in vivo effects of SU6668 on growth, angiogen- esis and microcirculation of chondrosarcomas by means of intravital microscopy.

\section{Methods}

\section{Animal model and cell lines}

Experiments were performed on 10 adult male severe combined immuno deficient mice (SCID, C.B-17/IcrCrlscid-BR, Charles River Laboratories Inc., Sulzfeld, Germany, 7 to 8 weeks old, 20 to $25 \mathrm{~g}$ body weight), following institutional guidelines approved by the local animal review board. All surgical procedures were performed in strictly aseptic conditions within a laminar flow unit (Merck Eurolab, Bruchsal, Germany) under deep anesthesia by an intra peritoneal injection of a mixture of ketamine (Ketanest ${ }^{\circledR}, 65$ mg/kg body weight, Pfizer, Karlsruhe, Germany), xylazine (Rompun ${ }^{\circledast}, 13 \mathrm{mg} / \mathrm{kg}$ body weight, Bayer, Leverkusen, Germany) and acepromazine (Sedastress $^{\circledast}, 2 \mathrm{mg} / \mathrm{kg}$ body weight, Medistar, Holzwickede, Germany).

The human chondrosarcoma cell line SW 1353 was obtained from ATCC (HTB-94, LGC Promochem GmbH, Wesel, Germany). Tumor cells $\left[1 \times 10^{7} / \mathrm{ml}\right]$ were injected subcutaneously into the left flank of each donor mouse and grown to a volume of 0.5 to $1.0 \mathrm{~cm}^{3}$. After sacrificing the donor mouse, the tumor was excised, cut into small chunks (volume 0.5-1.0 $\mathrm{mm}^{3}$ ) in Dulbecos Modified Eagle's Medium (DMEM, ) at $4^{\circ} \mathrm{C}$, and implanted into the recipient mouse in the following manner:

Surgical preparation of the cranial window was performed as described in detail elsewhere [14]. Briefly described, the scalp of the mouse was shaved and surgically excised in an oval area to expose the frontal and parietal bone. The periosteum was removed and an oval cavity of approximately 2 by 1 by $0.5 \mathrm{~mm}$ was milled into the calvaria by eliminating parts of the external tabula of the calvaria including the spongious bone underneath. Then one small chunk (approx. 0.5-1.0 $\mathrm{mm}^{3}$ ) of the human chondrosarcoma SW 1353 was implanted into the cavity. To prevent dehydration or mechanical damage to the tumors, the preparation was sealed with a glass cover slip and bone cement made of a mixture of ethyl cyanoacrylate glue (Pattex ${ }^{\circledR}$ Blitz Kleber, Henkel, Germany) and GC Ostron ${ }^{\circledR}$-Powder (methyl methacrylate polymer, GC Europe, Belgium).

The animals were housed individually in special filter cages to maintain aseptic conditions and to prevent mutual damage to the cranial window. The animals were provided with sterile standard pellet food and water ad libitum.

\section{SU6668 treatment}

SU6668 was provided by Sugen Inc. (San Francisco, California, USA) and was dissolved in a carboxymethylcellu- 
lose (CMC)-based vehicle at $50 \mathrm{mg}$ SU6668 per $\mathrm{ml}$ vehicle. Five animals each were treated by subcutaneous injection of $250 \mathrm{mg} / \mathrm{kg}$ body weight SU6668 (SU6668, n $=5$ ) or the equivalent amount of the (CMC)-based vehicle alone (Control, $\mathrm{n}=5$ ) every 48 hours. The chosen SU6668 dosage is in accordance with the dosages for animal trials reported in the literature and represents the lower range of dosages that were shown to be effective against primary tumors $[9,10,13,15,16]$. Treatment started on day 7 after tumor implantation and continued until termination of the experiments on day 28 after tumor implantation.

\section{Intravital microscopy}

For intravital microscopy, mice were anesthetized and positioned on a custom made stereotactic device.

In the first week after tumor implantation, mice were investigated daily under epi-illumination with a stereotactic microscope (Leica $\mathrm{MZ7}_{5}$, Leica, Germany) employing a 5 to 40 fold magnification. At 24-hour intervals, the first appearance of (i) hemorrhage, (ii) the first appearance of newly formed blood vessels entering the implanted tumor tissue, and (iii) the onset of perfusion in these newly formed vessels were determined.

Intravital fluorescence video microscopy was performed using an epi-illumination fluorescence microscope unit (Leica, Germany) equipped with a 4x (EF 4/0.12, Leitz, Wetzlar, Germany) and 40x (Zeiss Achroplan 40x/0.75 w, Carl Zeiss, Germany) objective on days 7, 14, 21, and 28 after tumor implantation. For off line analysis, regions of interest were recorded on video tapes using a S-VHS videocassette recorder (AG-7350, Panasonic, Japan) at a rate of 50 frames/s and a digital camera (Kappa CF 8/1, Kappa Opto-electronics, Germany).

Using an adequate fluorescence filter set for green light (bandpass 515-560 nm), the intravenous injection of the plasma marker fluorescein isothiocyanate (FITC)-labeled dextran (Sigma, St. Louis, MO, FITC-Dextran, FD 2000S, molecular weight $2.000 .000 ; 0.1 \mathrm{ml}$ of a $5 \%$ solution in $0.9 \% \mathrm{NaCl}$ ) enabled the observation of tumor microcirculation.

\section{Off-line analysis of tumor growth and microhemodynamics} Tumor growth was determined off-line by measuring its two-dimensional surface area in $\mathrm{mm}^{2}$ from standardized digital photographs of the cranial window preparation at 10-fold magnification on days 7, 14, 21, and 28 after implantation using a computer-based analysis program (AnalySIS ${ }^{\circledast}$ V3.0, Soft Imaging System, Münster, Germany).

The functional microvessel density (FVD) was determined as the length of all perfused microvessels within a tumor in relation to the two-dimensional surface area of the tumor in $\mathrm{mm} / \mathrm{mm}^{2}$ indicated by the fluorescence of FITClabeled dextran in all perfused vessels. Recordings on video tape for off-line analysis of the FVD were made for $15 \mathrm{~s}$, each. Off-line analysis was performed using a computer-based image analysis program (CapImage ${ }^{\circledast}$, Engineering Office Dr. Zeintl, Heidelberg, Germany).

\section{Histopathologic and immunohistochemical assessment}

The mice were sacrificed on day 28 after tumor implantation and the tumors were immediately excised along with the surrounding tissue of the calvaria and the brain for further histopathologic and immunohistochemical investigations. Tissue samples were fixed for 24-48 hours by immersion in $4 \%$ formalin solution. After decalcification of the bone in ethylene-diaminetetraacetic acid for 2 weeks, samples were embedded in paraffin and sliced into three- $\mu \mathrm{m}$ serial sections for Hematoxylin-Eosin staining and five- $\mu \mathrm{m}$ serial sections for immunohistochemistry.

To identify endothelial cells, immunohistochemistry for CD31 (PECAM-1) was performed. After deparaffinization, sections were pretreated with Retrievagen $A^{\circledR}$ solution (Cat. No. 550524, BD Pharmingen, Basel, Switzerland) for antigen retrieval at $90^{\circ} \mathrm{C}$ for 10 minutes. Tissue sections were placed into $0.3 \% \mathrm{H}_{2} \mathrm{O}_{2}$ in tris buffered saline (TBS) and 100\% goat serum afterwards to block endogenous peroxidase and unspecific binding, respectively. Tissue samples were incubated overnight at $4{ }^{\circ} \mathrm{C}$ with the primary antibody for CD31 (anti-mouse CD31 from rat, Cat. No. 550274, BD Pharmingen, Basel, Switzerland) or with mouse IgG for negative controls. Subsequently, the secondary antibody (biotinylated anti-rat IgG from goat, 1:1000, Cat. No. RPN1005, Amersham Biosciences, Otelfingen, Switzerland) and Streptavidin-horadish peroxidase conjugate (Cat. No. RPN1231, Amersham Biosciences, Otelfingen, Switzerland) were applied to the sections. Sections were then stained with 3-Amino-9-ethylcarbazole substrate (AEC, Cat. No. A6926, Sigma, Buchs, Switzerland), counterstained with hematoxylin and mounted with AquaTex.

\section{Statistics}

All numerical data are presented as median with 25\% and $75 \%$ quartiles. Using the software program SigmaStat ${ }^{\circledR}$ for Windows (Version 2.03, SPSS, Chicago, IL), data were analyzed statistically. The Mann-Whitney rank sum test was applied for pair wise comparison procedures. Differences were considered significant at $\mathrm{p}<0.05$.

\section{Results}

The first newly formed vessels in all tumors were observed 6 days after implantation. Vessel formation was followed by a rapid onset of perfusion within 24 hours. Intravital 
microscopy showed that the origin of angiogenic sprouting was from vessels located within the surrounding bone.

In Controls, functional vessel density (FVD) increased between day 7 and day 14 after tumor implantation, reaching a constant plateau between days 14 and 28 (Fig. 1a). After the initiation of therapy on day 7 after tumor implantation, FVD continuously decreased over the period of investigation with SU6668 (day 28: $5.5 \mathrm{~mm} /$ $\mathrm{mm}^{2}(5.1 / 6.3)$ vs. day $7: 7.8 \mathrm{~mm} / \mathrm{mm}^{2}(6.7 / 8.3)$; Fig. 1a). On day 28 after tumor implantation, FVD was significantly smaller with SU6668 compared to Controls (5.5 $\mathrm{mm} / \mathrm{mm}^{2}$ (5.1/6.3) vs. $8.6 \mathrm{~mm} / \mathrm{mm}^{2}$ (8.1/8.9); Fig. 1a).

Tumor growth was identical in all animals until day 7 after tumor implantation. In Controls, the two-dimen-

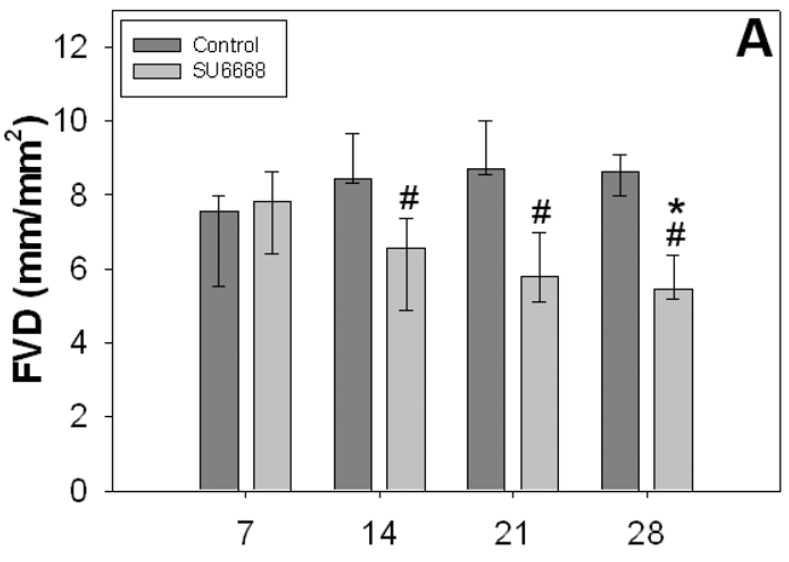

days after tumor implantation

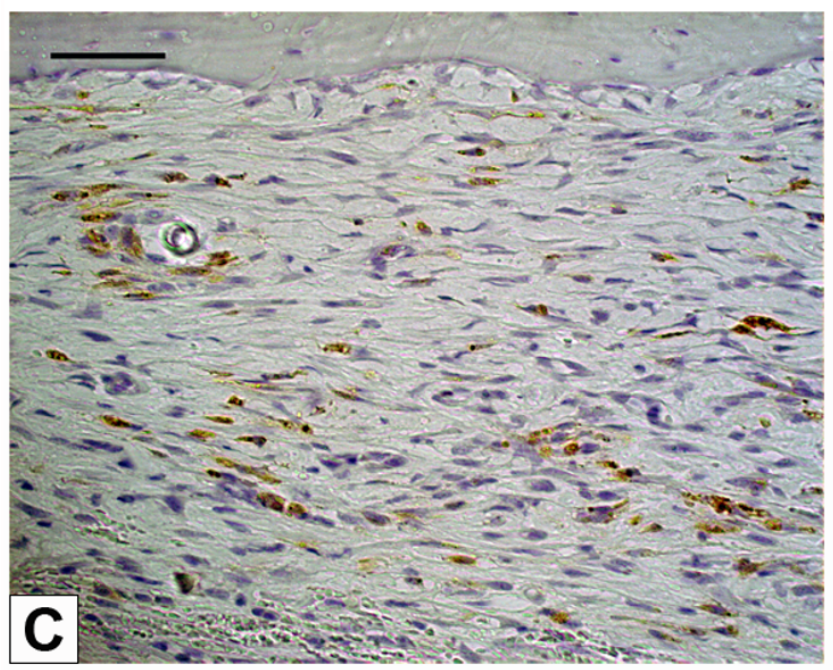

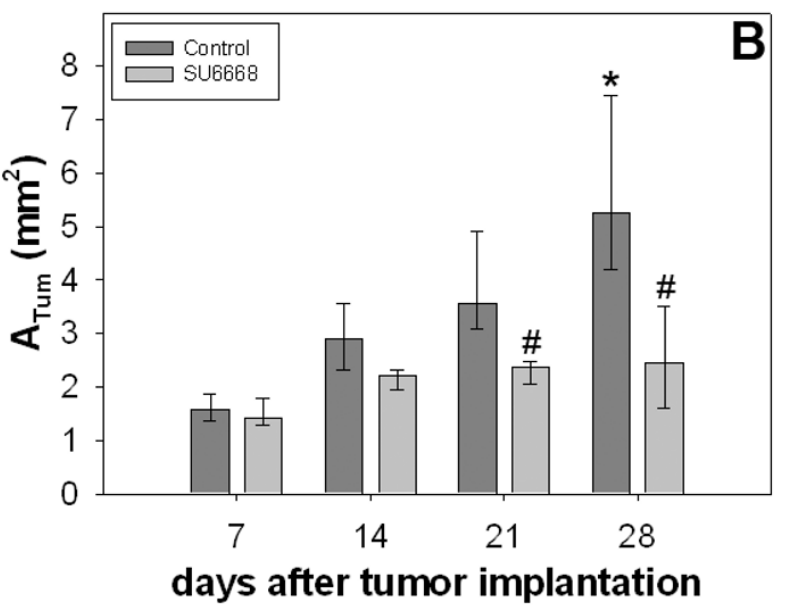

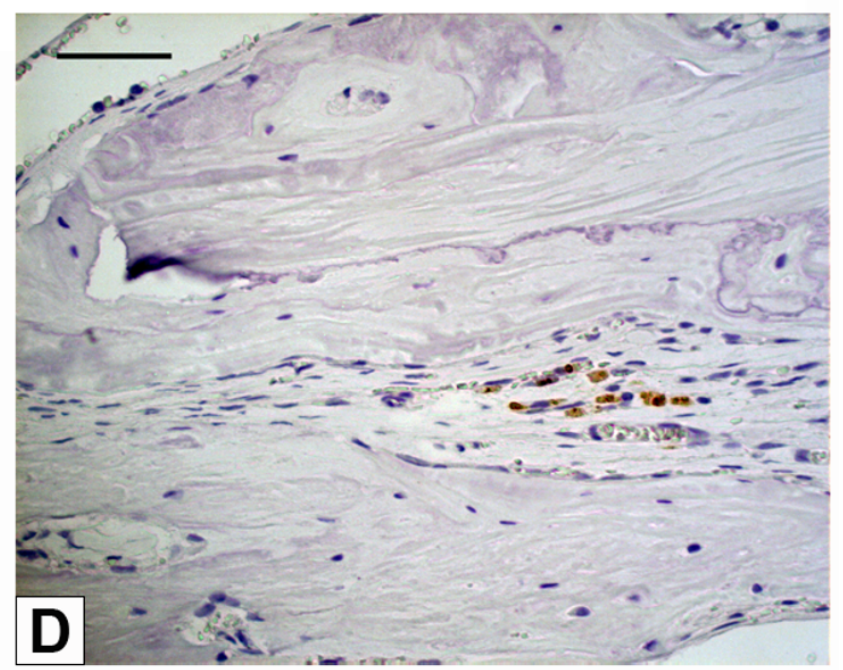

\section{Figure I}

A: Functional vessel density, time course. $Y$-axis: functional vessel density (FVD in $\mathrm{mm} / \mathrm{mm}^{2}$ ), $\mathrm{x}$-axis: time in days after tumor implantation. SU6668 induced a regression of functional vessel density (FVD), while FVD in Controls increased between day 7 and 14 after tumor implantation, remaining constant thereafter. B: Tumor surface, time course. Y-axis: tumor surface $\left(A_{T U M}\right.$ in $\mathrm{mm}^{2}$ ), $\mathrm{x}$-axis: time in days after tumor implantation. In Controls, tumor surface continuously increased over the period of investigation. In contrast to this, SU6668 induced a growth arrest of chondrosarcomas beginning on day 14 after tumor implantation. A and B: Treatment started on day 7 after tumor implantation. Results are given as median with $25 \%$ quartiles and 75 $\%$ quartiles. Mann-Whitney rank sum test, \#p $<0.05$ versus Control, ${ }_{p} p<0.05$ versus day 7 after tumor implantation. $\mathbf{C}$ and D: Immunohistochemistry for CD3I (PECAM-I) on day 28 after tumor implantation confirmed the results of the in vivo quantification of the functional vessel density with intravital microscopy. Untreated Controls showed uniformly distributed CD3 I positive cells (brown) within the implanted tumors (C). In SU6668 treated tumors, CD3I positive cells were rare and only detectable in peripheral areas of the tumor residues (D). Scale bars represent $50 \mu \mathrm{m}$. 
sional tumor surface $\left(\mathrm{A}_{\text {Tum }}\right)$ continuously increased over the period of investigation reaching $5.3 \mathrm{~mm}^{2}$ (4.4/7.2) on day 28 after tumor implantation (Fig. 1b). After initiation of the treatment on day 7 after tumor implantation, in SU6668 $\mathrm{A}_{\text {Tum }}$ increased until day 14 after tumor implantation. Subsequently to day 14 after tumor implantation, a growth arrest of chondrosarcomas occurred in SU6668. On day 28 after tumor implantation, the $\mathrm{A}_{\text {Tum }}$ was 2.5 $\mathrm{mm}^{2}$ (1.9/3.4) in SU6668, thus being statistically smaller than in Controls, where $A_{\text {Tum }}$ was $5.3 \mathrm{~mm}^{2}$ (4.4/7.2; Fig. $1 b)$.

The comparison of the time courses of FVD and $\mathrm{A}_{\text {Tum }}$ demonstrated that in SU6668, $\mathrm{A}_{\text {Tum }}$ had still increased in the first week after therapy initiation, while FVD was already regressive over the same time period of investigation.

Histologically, typical signs of a malignant tumor were observed in Controls on day 28 after tumor implantation. In accordance to in vivo findings, the volume of chondrosarcomas increased significantly compared to the small chunks (volume $0.5-1.0 \mathrm{~mm}^{3}$ ) that were initially implanted (Fig. 2a). Large tumor masses had grown above, below, and into the calvaria. Histology revealed extensive infiltration and resorption of the adjoining bone as signs for typical growth behavior of malignant tumors. However, infiltration into the underlying brain was not observed on day 28 after tumor implantation (Fig. 2a-c). In contrast to this, the volume of chondrosarcomas in SU6668 remained at a much smaller level compared to Controls. Furthermore, tumors showed signs of regression in SU6668. On day 28 after tumor implantation, there was not one large formation of any solid tumor, but residual chondrosarcoma cells were observed embedded in fibrous tissue that filled large parts of the cranial window preparation (Fig. $2 \mathrm{~d}-\mathrm{f}$ ). In regions of previous bone resorption, regenerative bone formation was observed (Fig. 2e). The immunohistochemical staining of endothelial cells confirmed the results of the in vivo quantification of the functional vessel density with intravital microscopy. Untreated tumors showed uniformly distributed CD31 positive endothelial cells. In contrast, tumors which were treated with SU6668 were only weekly positive for CD31 (Fig. 1c-d).

Injections of SU6668 or of the CMC-vehicle alone were well tolerated, as no differences in animal behavior or loss of weight were observed.

\section{Discussion}

Due to the insufficient response of chondrosarcomas to chemotherapy and ionizing radiation, current curative therapy of these tumors is usually limited to surgery. As angiogenesis is essential for tumor growth, the selective destruction of tumor feeding and draining blood vessels represents a promising strategy for overcoming the therapeutic limitations of malignant primary bone tumors.

The small molecule receptor tyrosine kinase inhibitor SU6668 was used as an antiangiogenic agent against the human chondrosarcoma SW1353, which was grown in a cranial window preparation in SCID mice. SU6668 mediates the simultaneous inhibition of the receptor-tyrosine kinase activity of vascular endothelial growth factor (VEGF), fibroblast growth factor (FGF), and plateletderived growth factor (PDGF), which are key mediators of tumor angiogenesis [9,15,17-19]. SU6668 has already shown encouraging antiangiogenic effects on some solid tumors [9-11]. However, the effects of SU6668 on primary bone tumors have only been described once for Ewing's sarcoma family tumors [13].

The present data demonstrate the effective anti-tumor action of SU6668. Treatment of SW1353 chondrosarcomas with SU6668 at $250 \mathrm{mg} / \mathrm{kg} / 48 \mathrm{~h}$ decreased the tumor size to $53 \%$ of the size in Controls on day 28 after tumor implantation. The time course of two-dimensional tumor surfaces demonstrated that only SU6668 induced a growth arrest of tumors starting on day 14 after tumor implantation. In Controls, at the same time a continuous increase of the tumor size was measured. The in vivo findings were confirmed by histology from tissues excised on day 28 after tumor implantation. In Controls, typical signs of the behavior of malignant tumor growth were observed while SU6668 treatment resulted in small residual tumors with the formation of scar tissue within the tumor. These results are consistent with previous findings on growth inhibition of tumor xenografts by SU6668: Laird et al. [15] found a slight increase in volumes of the human epidermoid tumor xenograft A431 within the first week after initiation of SU6668 treatment resulting in a growth arrest of these tumors thereafter. Marzola et al. [10] described a $60 \%$ growth inhibition of HT-29 colon carcinomas treated with SU6668 compared to untreated Controls.

The anti-tumor activity of SU6668 has been attributed to its antiangiogenic potential arising from its inhibitory effect on the receptor-tyrosine kinase activity of VEGF, FGF, and PDGF. Inhibition of the downstream action of these angiogenic receptors has been shown to induce endothelial cell apoptosis $[9,20]$. Furthermore, Laird et al. [9] showed that endothelial cell apoptosis preceded tumor cell apoptosis. The authors concluded that SU6668 treatment initiates vessel death by apoptosis, which in turn leads to subsequent tumor cell death. In the present study, SU6668 significantly reduced functional vessel density of tumors by $37 \%$ compared to Controls, demonstrating the antiangiogenic potential of SU6668. A comparison of the time courses of functional vessel density 


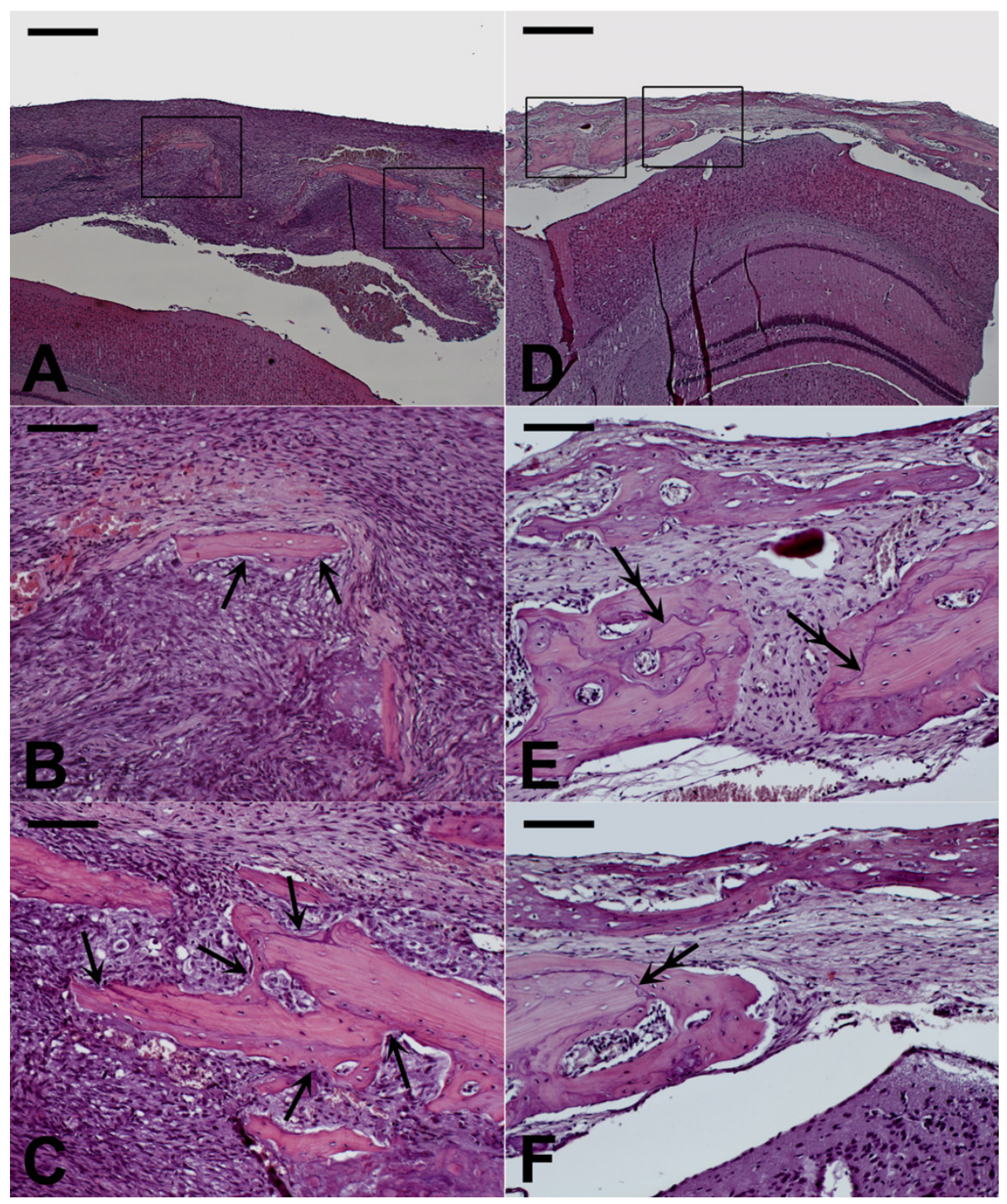

\section{Figure 2}

SW I 353 chondrosarcoma at day 28 after tumor implantation, hematoxylin-eosin stained cross section from representative tissue samples of the cranial window preparation. A, D: 4-fold magnification, scale bar $500 \mu \mathrm{m}$. B, C, E, F: 20-fold magnification, scale bar $100 \mu \mathrm{m}$. In Controls, the overview magnification shows a large tumor formation with high cellular density (A).

Enlarged cutaway views (B, C) from the framed regions show high cellular density and destructive tumor growth with resorption of the surrounding bone (arrows, tips pointing to resorption lacunae). In SU6668, tumor size was markedly reduced (D). Enlarged cutaway views (E, F) from the framed regions show fibrous "scar" tissue with residual tumor cells and low cellular density and regenerative bone formation at sites of previous bone resorption (double arrows, distal tip pointing to former resorption front, proximal tip pointing to new mineralization front).

and tumor size demonstrated that SU6668 primarily induced a reduction in tumor vascularization directly after initiation of this therapy. This was followed by growth arrest of the implanted chondrosarcomas starting on day 14 after tumor implantation. In accordance with findings by Laird et al. [9], and analogous to findings from a previous study where Celecoxib-induced inhibition of the functional vessel density caused a reduced growth of the lung carcinoma A 549 implanted into a cranial window preparation [21], we conclude that an underlying mechanism of the anti-tumor effect of SU6668 is the antiangiogenic potential resulting in a reduction in blood vessels that feed and drain tumors, which in turn halts tumor progression. 
Although the tumor vasculature is thought to be little prone to acquiring drug resistance $[3,22,23]$ recurrence of tumor growth after an initial period of suppression during long-term antiangiogenic therapy has also been described [24-26]. Acquired drug resistance has predominantly been described for drugs only interfering with a single angiogenic pathway [24-26]. As SU6668 does not target just one signaling pathway but inhibits signal transduction through the growth factor receptors VEGFR2, PDGFb2 and FGFR1, drug resistance is less likely to occur [24].

\section{Conclusion}

The data presented demonstrate that SU6668 is a potent inhibitor of tumor growth of chondrosarcomas in vivo. The anti-tumor effects can be attributed to the antiangiogenic effects of the substance, which are mediated by the inhibition of the receptor tyrosine kinases Flk-1/KDR (VEGR2), PDGFRbeta and FGFR1. Antiangiogenic therapy using the application of SU6668 appears to be a promising way to expand the therapeutic options in chondrosarcoma therapy. However, long-term studies must be performed to elucidate the effects of a long-term treatment such as potential side effects, the development of drug resistance or relapse of tumor growth.

\section{Competing interests}

The author(s) declare that they have no competing interests.

\section{Authors' contributions}

FMK participated in designing the study, carried out the animal experiments, the histology and immunohistochemistry, interpreted the data, and drafted the manuscript. MMG and VE participated in designing the study and revising the manuscript critically. AA and PEH established the SW 1353 cell cultures and tumor cell suspensions and participated in revising critically the manuscript. EB carried out data acquisition from the in vivo experiments. AS conceived, coordinated, and designed the study, performed the statistical analysis, interpreted the data, and drafted the manuscript. All of the authors have read and approved the final manuscript.

\section{Acknowledgements}

We thank K. Goetzke and R. Föhr for their technical support on the histological stainings; B. Hillenbrand and M. Schwandt for their support during data acquisition. This study was generously supported by a research grant (F.02.0015) of the MWFK Ba-Wue (Ministry for Science, Research, and Art of the State of Baden-Wurttemberg, Germany) to AS.

\section{References}

I. Folkman J: Anti-angiogenesis: new concept for therapy of solid tumors. Ann Surg 1972, 175:409-416.

2. Carmeliet $P:$ Mechanisms of angiogenesis and arteriogenesis. Nat Med 2000, 6:389-395.

3. Singh RP, Agarwal R: Tumor angiogenesis: a potential target in cancer control by phytochemicals. Curr Cancer Drug Targets 2003, 3:205-217.
4. Tosetti F, Ferrari N, De Flora S, Albini A: Angioprevention': angiogenesis is a common and key target for cancer chemopreventive agents. FASEB J 2002, 16:2-14.

5. Keshet E, Ben Sasson SA: Anticancer drug targets: approaching angiogenesis. J Clin Invest 1999, 104:1497-I50I.

6. Abdollahi A, Lipson KE, Han X, Krempien R, Trinh T, Weber KJ, Hahnfeldt P, Hlatky L, Debus J, Howlett AR, Huber PE: SU54I6 and SU6668 attenuate the angiogenic effects of radiationinduced tumor cell growth factor production and amplify the direct anti-endothelial action of radiation in vitro. Cancer Res 2003, 63:3755-3763.

7. Bischof M, Abdollahi A, Gong P, Stoffregen C, Lipson KE, Debus JU, Weber KJ, Huber PE: Triple combination of irradiation, chemotherapy (pemetrexed), and VEGFR inhibition (SU5416) in human endothelial and tumor cells. Int $J$ Radiat Oncol Biol Phys 2004, 60:1220-1232.

8. Huber PE, Bisch of M, Jenne J, Heiland S, Peschke P, Saffrich R, Grone HJ, Debus J, Lipson KE, Abdollahi A: Trimodal cancer treatment: beneficial effects of combined antiangiogenesis, radiation, and chemotherapy. Cancer Res 2005, 65:3643-3655.

9. Laird AD, Christensen JG, Li G, Carver J, Smith K, Xin X, Moss KG, Louie SG, Mendel DB, Cherrington JM: SU6668 inhibits Flk-II KDR and PDGFRbeta in vivo, resulting in rapid apoptosis of tumor vasculature and tumor regression in mice. FASEB J 2002, 16:68I-690.

10. Marzola P, Degrassi A, Calderan L, Farace P, Crescimanno C, Nicolato E, Giusti A, Pesenti E, Terron A, Sbarbati A, Abrams T, Murray L, Osculati $F$ : In vivo assessment of antiangiogenic activity of SU6668 in an experimental colon carcinoma model. Clin Cancer Res 2004, 10:739-750.

II. Garofalo A, Naumova E, Manenti L, Ghilardi C, Ghisleni G, Caniatti M, Colombo T, Cherrington JM, Scanziani E, Nicoletti MI, Giavazzi R: The combination of the tyrosine kinase receptor inhibitor SU6668 with paclitaxel affects ascites formation and tumor spread in ovarian carcinoma xenografts growing orthotopically. Clin Cancer Res 2003, 9:3476-3485.

12. Griffin RJ, Williams BW, Wild R, Cherrington JM, Park H, Song CW: Simultaneous inhibition of the receptor kinase activity of vascular endothelial, fibroblast, and platelet-derived growth factors suppresses tumor growth and enhances tumor radiation response. Cancer Res 2002, 62:1702-1706.

13. Dalal S, Berry AM, Cullinane CJ, Mangham DC, Grimer R, Lewis IJ, Johnston C, Laurence V, Burchill SA: Vascular endothelial growth factor: a therapeutic target for tumors of the Ewing's sarcoma family. Clin Cancer Res 2005, I I:2364-2378.

14. Klenke FM, Merkle T, Fellenberg J, Abdollahi A, Huber PE, Gebhard MM, Ewerbeck V, Sckell A: A novel model for the investigation of orthotopically growing primary and secondary bone tumours using intravital microscopy. Lab Anim 2005, 39:377-383.

15. Laird AD, Vajkoczy P, Shawver LK, Thurnher A, Liang C, Mohammadi $M$, Schlessinger J, Ullrich A, Hubbard SR, Blake RA, Fong TA, Strawn LM, Sun L, Tang C, Hawtin R, Tang F, Shenoy N, Hirth KP, McMahon $G$, Cherrington: SU6668 is a potent antiangiogenic and antitumor agent that induces regression of established tumors. Cancer Res 2000, 60:4152-4160.

16. Machida S, Saga Y, Takei Y, Mizuno I, Takayama T, Kohno T, Konno $R$, Ohwada M, Suzuki M: Inhibition of peritoneal dissemination of ovarian cancer by tyrosine kinase receptor inhibitor SU6668 (TSU-68). Int J Cancer 2005, I I 4:224-229.

17. Gale NW, Yancopoulos GD: Growth factors acting via endothelial cell-specific receptor tyrosine kinases: VEGFs, angiopoietins, and ephrins in vascular development. Genes Dev 1999, 13:1055-1066.

18. Klint P, Claesson-Welsh L: Signal transduction by fibroblast growth factor receptors. Front Biosci 1999, 4:DI65-DI77.

19. Rosenkranz S, Kazlauskas A: Evidence for distinct signaling properties and biological responses induced by the PDGF receptor alpha and beta subtypes. Growth Factors 1999, 16:201-216.

20. Erber R, Thurnher A, Katsen AD, Groth G, Kerger H, Hammes HP, Menger MD, Ullrich A, Vajkoczy P: Combined inhibition of VEGF and PDGF signaling enforces tumor vessel regression by interfering with pericyte-mediated endothelial cell survival mechanisms. FASEB J 2004, 18:338-340.

21. Klenke FM, Gebhard MM, Ewerbeck V, Abdollahi A, Huber PE, Sckell $A$ : The selective Cox-2 inhibitor Celecoxib suppresses angio- 
genesis and growth of secondary bone tumors: an intravital microscopy study in mice. BMC Cancer 2006, 6:9.

22. Kerbel RS: Inhibition of tumor angiogenesis as a strategy to circumvent acquired resistance to anti-cancer therapeutic agents. Bioessays 1991, 13:31-36.

23. Boehm T, Folkman J, Browder T, O'Reilly MS: Antiangiogenic therapy of experimental cancer does not induce acquired drug resistance. Nature 1997, 390:404-407.

24. Casanovas O, Hicklin DJ, Bergers G, Hanahan D: Drug resistance by evasion of antiangiogenic targeting of VEGF signaling in late-stage pancreatic islet tumors. Cancer Cell 2005, 8:299-309.

25. Klement G, Baruchel S, Rak J, Man S, Clark K, Hicklin DJ, Bohlen P, Kerbel RS: Continuous low-dose therapy with vinblastine and VEGF receptor-2 antibody induces sustained tumor regression without overt toxicity. I Clin Invest 2000, I05:R I5-R24.

26. Ton NC, Jayson GC: Resistance to anti-VEGF agents. Curr Pharm Des 2004, 10:51-64.

\section{Pre-publication history}

The pre-publication history for this paper can be accessed here:

http://www.biomedcentral.com/1471-2407/7/49/prepub

Publish with Biomed Central and every scientist can read your work free of charge

"BioMed Central will be the most significant development for disseminating the results of biomedical research in our lifetime. "

Sir Paul Nurse, Cancer Research UK

Your research papers will be:

- available free of charge to the entire biomedical community

- peer reviewed and published immediately upon acceptance

- cited in PubMed and archived on PubMed Central

- yours - you keep the copyright 